\title{
The tip-of-the-tongue state bias permeates unrelated concurrent decisions and behavior
}

\author{
Anne M. Cleary ${ }^{1} \cdot$ Andrew M. Huebert ${ }^{1} \cdot$ Katherine L. McNeely-White ${ }^{1}$ \\ Published online: 13 January 2020 \\ (C) The Psychonomic Society, Inc. 2020
}

\begin{abstract}
The tip-of-the-tongue (TOT) state — the feeling of being near accessing an as yet inaccessible word from memory —is associated with cognitive bias. For example, prior work has shown that TOTs are associated with a bias toward inferring positive qualities of the unretrieved information. People are biased during TOTs to indicate that the unretrieved target has a greater likelihood of being positively valenced and to have been associated with a higher value number earlier in the experiment. Additionally, when the TOT is for a pictured person's name, that person is judged to be more likely to be ethical. The present study demonstrates that the TOT positivity bias extends to unrelated concurrent decisions and behavior. In Experiment 1, participants reported a greater inclination to take an unrelated gamble during TOTs than non-TOTs. Experiment 2 demonstrated the concurrent nature of this spillover effect. The TOT bias toward a greater inclination to gamble significantly diminished with a 10-second delay between the time of reporting the TOT state and the time to report the inclination. Finally, Experiment 3 showed that the increased inclination to want to take a gamble during TOTs translated to actual gambling behavior. Participants chose to gamble for points more often during TOTs than non-TOTs.
\end{abstract}

Keywords Metamemory $\cdot$ Judgment $\cdot$ Heuristics $\cdot$ Memory $\cdot$ Tip of the tongue $\cdot$ Risk-taking $\cdot$ Gambling

The tip-of-the-tongue (TOT) state - the feeling of being on the verge of accessing an unrecalled word-has been the subject of scientific investigation for decades (e.g., Brown, 1991, 2012; Brown \& McNeill, 1966; Gollan \& Brown, 2006; Schwartz, 2002). Most research over the years has aimed to understand the TOT state's underlying mechanisms (e.g., Burke, MacKay, Worthley, \& Wade, 1991; Gollan \& Brown, 2006; Koriat \& Lieblich, 1974; Rastle \& Burke, 1996; Schwartz, 1999, 2001, 2008; Schwartz \& Metcalfe, 2011; Schwartz \& Smith, 1997; Schwartz, Travis, Castro, \& Smith, 2000). However, the TOT state has recently been discovered to be associated with a form of cognitive bias (e.g., Cleary, 2018; Cleary \& Claxton, 2015). One aspect of this bias is that TOTs are associated with inferences of positivity regarding the unretrieved target information (Cleary, 2018) - an aspect that will henceforth be referred to as the TOT positivity bias. The present study examined whether the TOT positivity bias extends to concurrent unrelated decisions, as opposed to

Anne M. Cleary

Anne.Cleary@colostate.edu

1 Department of Psychology, Colorado State University, 1976 Campus Delivery, Fort Collins, CO 80523-1876, USA just decisions about the unretrieved target information, and if so, whether this translates to effects on actual behavior.

\section{History of the TOT bias}

The first indication of the existence of a TOT state bias was an inadvertent discovery in a study that was actually aimed at investigating a different phenomenon known as recognition without identification (Cleary, 2006). In the study, answers to general-knowledge questions were presented as sequential to-be-remembered study items on a study list. Following the study list, participants were tested with general-knowledge questions, half of which had had their answers studied and half of which had not. The interest was in the questions that went unanswered, to determine if participants could have a sense that the answer was studied when the answer itself could not be retrieved from memory (the recognition without identification effect). However, in an experiment in which participants were also asked to indicate the presence versus absence of a TOT state for the unretrieved target answer, it was discovered that participants thought it more likely that the unretrieved target answer had been on the study list during reported TOT states than during reported non-TOT states. 
This was despite the fact that studying an answer actually led to a decrease in the likelihood of a TOT, suggesting that the effect was a bias. It was as if participants were inferring from the presence of a TOT state a greater likelihood that the unretrieved target answer was studied. This pattern was replicated in follow-up studies that examined TOT reports in the context of recognition without identification experiments (e.g., Cleary, Konkel, Nomi, \& McCabe, 2010; Cleary \& Reyes, 2009; Cleary \& Specker, 2007), then reviewed by Cleary, Staley, and Klein (2014) with a call for future research on whether TOT states are associated with other types of inferences, besides whether the unretrieved target word was studied.

Cleary and Claxton (2015) carried out the first study to examine whether other forms of cognitive bias are associated with TOT reports. They reported several experiments demonstrating that during reported TOT states (relative to reported non-TOT states during instances of target word retrieval failure), participants inferred a greater likelihood that the unretrieved target word had attributes that tend to characterize high fluency or accessibility. For example, during TOT states, participants were more likely to infer a greater likelihood that that the unretrieved target word had appeared earlier in the experiment in a darker, clearer font, even though there was no such association with the probability of a TOT report, and even among unretrieved targets that had not been presented earlier in the experiment. Participants were also analogously more likely to infer a greater likelihood that the unretrieved target word had appeared earlier in the experiment in a larger font size, even though there was no such association with the probability of a TOT report, and even among unretrieved targets that had not been presented earlier in the experiment. Finally, participants were also more likely to infer a greater likelihood during TOT states that the unretrieved target word was a higher frequency word, despite no greater likelihood of reporting a TOT state for high-frequency words relative to low-frequency words. Cleary and Claxton suggested that a potential reason for this inference might be that, because the TOT state is defined as feeling on the verge of retrieving the currently inaccessible target word, participants assume from the presence of this state that those unretrieved words must be more fluent or accessible than the unretrieved words that do not elicit a TOT state.

\section{The TOT positivity bias}

Cleary (2018) discovered that TOT states are associated with a bias toward inferring positive qualities or characteristics about the unretrieved target. Relative to non-TOT states, during TOT states, participants inferred a greater likelihood that the unretrieved target was positively valenced as opposed to negatively valenced (Experiment 1), and that it was more likely to be associated with a higher value number earlier in the experiment (Experiment 2), despite that TOTs were not any more likely for targets studied with high-value numbers as opposed to with low-value numbers. In addition, when the reported TOT state was for a pictured person's name, participants inferred a greater likelihood that the pictured person was ethical relative to when the unnamable person's face did not elicit a TOT state (Experiment 3).

Cleary (2018) demonstrated that this TOT bias pattern was not due to an increased inclination to simply give higher ratings (or select higher numbers) on any continuum during TOT states. In a variation of the experiment in which participants exhibited a TOT positivity bias toward indicating a greater likelihood that a pictured person was ethical, Cleary found that reversing the scale yielded the same TOT positivity bias. That is, when, on the scale of zero to 10 , zero meant "definitely ethical" and 10 meant "definitely unethical," participants gave lower ratings when in a TOT state than when not. They did so to the same extent as when the scale was reversed, such that 10 meant "definitely ethical" and zero meant "definitely unethical." Thus, the TOT bias was indeed a positivity bias. Regardless of the nature of the scale, participants were more inclined to judge a pictured person as being more ethical when in a TOT state for that person's name than when not.

It may seem counterintuitive that a TOT state would be associated with positive, rather than negative, valence. After all, TOTs are often anecdotally described as frustrating (Brown, 2012; Schwartz, 2002), and in their seminal article, Brown and McNeill (1966) used terms like "agony" and "mild torment" to describe the experience. Perhaps for this reason, previous work on TOT emotional components has tended to focus exclusively on negative versus neutral valence (e.g., Schwartz, 2010), and perhaps because the assumption about negative valence is wrong, no consistent effects of these comparisons have emerged (D'Angelo \& Humphreys, 2012).

Upon closer inspection, it is perhaps not as counterintuitive that TOTs would be associated with positive valence as it seems at first glance. Another phrase used by Brown and McNeill was "on the brink of a sneeze," which might be viewed as a more positive-seeming description of the feeling of the TOT, insofar as it implies that the relief of retrieving the answer may be right around the corner. Furthermore, Brown (2012) suggests that it is possible that TOTs could be accompanied by excitement, which might also be viewed as a positive emotion. In support of the idea that TOTs may be accompanied by excitement, TOTs do appear to be correlated with momentary changes in nervous system activity that relate to excitement. Ryals, Reynolds, Patton, and Cleary (2018) found increased pupil dilation during reported TOTs than non-TOTs. Increased pupil dilation can indicate increased arousal, which is consistent with the idea that TOTs are accompanied by excitement. Also consistent with the idea that TOTs may be felt as excitement is the finding, by Metcalfe, Schwartz, and 
Bloom (2017), that TOTs are associated with increased curiosity and motivation to discover the identity of the as yet unretrieved target. When there are limited opportunities to find out the as yet unknown answer to a question, participants are more inclined to use those limited opportunities for discovery during TOT than non-TOT states.

Cleary (2018) suggests that the TOT feeling may be experienced as a sign of "getting warmer" in the hunt for the sought-after information. Put differently, TOTs may be experienced as an indication of the presence of something relevant in the knowledge base, rather than nothing at all, which may be perceived as positive. This notion is consistent with the theoretical perspective put forth by Gollan and Brown (2006), in which they argue that rather than being viewed as instances of retrieval failure, TOTs should be viewed as instances of retrieval success, because they are indicative of the presence of relevant knowledge - a form of success rather than failure. Insofar as a TOT is an indication of a form of success (the answer is there in the knowledge base, as opposed to not known at all), it may make sense that its valence would be positive, rather than negative, at least upon initially feeling it.

Along these lines, Cleary (2018) related the TOT positivity bias to the "warm glow" heuristic (e.g., Monin, 2003), suggesting that the feeling accompanying the TOT state may be akin to a "warm glow" or a momentary positive feeling. Cleary argues that this momentary positive feeling is what leads to inferences that the unretrieved information has positive qualities or characteristics. The momentary positive feeling leads to these inferences.

\section{Does the TOT positivity bias permeate concurrent decisions?}

If TOTs are associated with a momentary bias toward positivity, does this positivity bias extend to concurrent decisions that must be made at the time of the TOT state? There is evidence that attributions based on one type or piece of information can influence concurrent unrelated decisions. The halo effect is one such piece of evidence (Morewedge \& Kahneman, 2010; Nisbett \& Wilson, 1977). The halo effect refers to the finding that one perceived quality or characteristic, such as a person's perceived attractiveness, can affect the perceiver's judgment about a completely unrelated quality. For example, a person perceived as attractive may also be perceived as more competent, more intelligent, or more kind. In this example, one positive quality seems to lead to inferences of other positive qualities, even though the other positive qualities should be viewed as unrelated to the first. Some have suggested that the mechanisms behind the halo effect are a type of priming effect, whereby thinking of one type of positive quality evokes the automatic activation of concepts that are associated with positive traits in general, affecting additional judgments that should be considered unrelated (Morewedge \& Kahneman, 2010).

Given the existence of the halo effect, it is conceivable that the TOT positivity bias is not limited to situations in which the judgments are about the unretrieved information itself; rather, like the halo effect, the TOT positivity bias may extend to decisions that happen to coincide in timing with the onset of the TOT experience. If, as Cleary (2018) suggests, TOT states are experienced in the moment as somewhat positive (perhaps as feelings of excitement or motivation), then such feelings might permeate other decisions that happen to be made in the aftermath of the TOT onset. ${ }^{1}$

To investigate this question, we deliberately sought out a type of decision that would be completely unrelated to the TOT experience itself or to the attempt at word retrieval. Toward this end, we sought out a decision type that would not ordinarily occur during attempts at word retrieval. At the same time, the decision would need to be one that could plausibly be affected by a momentary feeling of positivity. For what type of completely unrelated decision might a momentary feeling of positivity exert a biasing influence?

One candidate type of decision is about whether or not to take an unrelated risk. An abundance of evidence suggests that one's current mindset primes concurrent judgments and decisions about risk-taking in ways that are biasing. That one's current mindset can be biasing regarding concurrent decisions is well known. One example is the aforementioned halo effect, which may result from automatic priming of positive concepts that affect one's current mindset in ways that bias judgments (e.g., Morewedge \& Kahneman, 2010). Another is the finding that mood induction (positive or negative) influences whether one retrieves positive or negative words from an earlier list (Teasdale \& Russell, 1983); an induced positive mindset leads to selective retrieval of positively valenced words, whereas an induced negative mindset leads to selective retrieval of negatively valenced words. Most relevant to the present purposes, similar types of priming-of-mindset effects have been shown to occur with risk-taking decisions and behavior (e.g., Payne, Brown-Iannuzzi, \& Loersch, 2016; Young, Wohl, Matheson, Baumann, \& Anisman, 2008). Most notably, Ludvig, Madan, and Spetch (2015) found that priming people with cue reminders of past wins led to increased risk-taking in the form of gambling. This evidence points toward the plausibility that a positive current mindset alone may prime increased risktaking behavior. Although to our knowledge no one has yet examined whether a temporary positive feeling can increase

\footnotetext{
${ }^{1}$ It is important to note that the present study does not speak to the issue of whether TOT states cause a feeling of a warm glow, or if a warm glow causes the perception of a TOT state. The direction of causality is currently unknown. We are only claiming that there is an existing association between TOT states and feelings of positivity, and that because of this association, biases that are related to the TOT state may permeate concurrent, unrelated decisions.
} 
risk-taking decisions, these priming studies point toward the possibility that it can. Thus, a risk-taking type of decision was chosen for the present study because (1) it would not ordinarily occur during word retrieval attempts and thus can be viewed as a completely unrelated decision to the attempt at word retrieval and to the unretrieved target information, and (2) it could plausibly be affected by the TOT positivity bias insofar as a positive mindset or disposition can potentially affect decisions about risk-taking.

Following from prior studies of risk-taking (e.g., Ludvig et al., 2015), the specific task used in the present study was a gambling task. Experiment 1 aimed to examine whether the presence of a TOT state would bias participants toward feeling a greater inclination to take an unrelated gamble. Following the prompt to indicate a TOT or non-TOT state, participants were prompted to indicate their inclination to take an unrelated gamble (a coin flip that would involve gaining or losing points depending on a win or a loss, with a decision to not gamble meaning neither gaining nor losing points). The interest was in whether participants would feel more inclined to take a gamble following TOT than non-TOT reports.

Experiment 2 was intended to follow up on Experiment 1's result to examine whether any spillover effect of the TOT positivity bias is indeed concurrent with the onset of the TOT state. If the TOT positivity bias is due to a momentary feeling associated with the onset of the TOT state, then it should not be long lived, though this has never been directly examined. To investigate this, Experiment 2 involved the same procedure as in Experiment 1, but compared prompting the rating of the inclination to gamble immediately versus 10 seconds following the TOT prompt.

Finally, Experiment 3 examined whether the TOT positivity bias would extend to actual behavior, as opposed to just the rated inclination to gamble versus not gamble. Experiment 3 followed the same procedure as Experiment 1, but participants had the opportunity to actually gamble or not gamble, with actual points won or lost. The interest was in whether participants would actually gamble more often immediately following TOTs than non-TOTs.

\section{Experiment 1}

Experiment 1 was aimed at determining whether the TOT bias is limited to decisions about the unretrieved information itself, or if the TOT bias extends to unrelated decisions that must be made concurrently, while or shortly after the person is experiencing the TOT. Toward this end, Experiment 1 served to examine whether the TOT bias would extend to judgments of one's current inclination to take an unrelated gamble (as that is not a decision about the unretrieved information).

\section{Method}

Participants Nineteen Colorado State University students participated in exchange for credit in a course. Past research on the TOT bias suggests that the bias tends to be a large effect that does not require a large number of participants. For example, Cleary (2006, Experiment 2) found the first TOT bias effect with 14 participants (as an unexpected finding in a study investigating another phenomenon). Indeed, based on the effect size obtained with Cleary and Claxton's (2015) pairedsamples $t$ test for the TOT bias among trials for which the target was not studied earlier in Experiment 1a (Cohen's $d=$ .73), a power level of .80 for a two-tailed test at an alpha level of .05 would require 17 participants.

Materials Stimuli were the 80 general-knowledge questions and their answers used by Cleary and Claxton (2015), which came from Nelson and Narens's (1980) norms. This subset was selected for continued relevance to modern-day participants.

Procedure The 80 general-knowledge questions appeared in a random order, each followed by a series of dialog box prompts. At the start of the experiment, participants were instructed on the nature the study. They were told that they would view a series of general-knowledge questions, for which they would be prompted to attempt to provide the answer. They were further instructed to indicate any instances in which they felt that they were in a tip-of-the-tongue state for the answer. As in prior research (e.g., Cleary, 2006; Cleary, 2018; Schwartz, 2001), a TOT state was defined as follows: "You feel as if it is possible that you could recall the target answer, and you feel as if its recall is imminent. It's as if the answer is on the 'tip of your tongue,' about to be recalled, but you simply cannot think of the word at the moment." Participants were then instructed that they would also be asked to make a judgment about whether they felt inclined to take an unrelated gamble. They were instructed as follows regarding the gambling judgment:

After indicating if you are experiencing a tip-of-thetongue state, you will be asked to judge your inclination to take a gamble versus not take a gamble. For this, you will be asked to give a rating on a scale of 0 (definitely not inclined to gamble) to 10 (definitely inclined to gamble). Choosing to gamble means hypothetically (in an imagined scenario) flipping a coin with a 50/50 probability of getting heads versus tails. Heads means you gain 10 points. Tails means you lose 10 points. Choosing not to gamble means you stay the same, neither winning nor losing points. You won't give an all-or- 
none judgment or actually take a gamble - just rate your momentary inclination toward gambling or not gambling in that moment, when asked.

Once beginning the experiment, the general-knowledge questions appeared in a random order. For each generalknowledge question (e.g., "What is the name of the inability to sleep?"), participants first attempted to answer the question by typing the answer (e.g., "insomnia") before pressing Enter, or by simply pressing Enter if they could not think of the answer. Participants were then prompted to indicate if they were experiencing a TOT state for the answer. Participants were then prompted to rate their inclination to take a gamble using a scale of zero (definitely not inclined to gamble) to 10 (definitely inclined to gamble), regardless of whether they reported being in a TOT state, and regardless of whether or not they had successfully identified the answer.

After this, participants were prompted with a second chance at identifying the answer and encouraged to guess on this attempt. Finally, as in prior research (Cleary, 2006; Cleary \& Claxton, 2015; Cleary et al., 2014), participants were prompted for partial information about unretrieved targets, such as the first letter, what the word sounds like, and the number of syllables in the word. Trials on which word retrieval failed but correct partial word information was identified were removed from consideration, as these could be considered instances of successful partial retrieval of the word, and the primary interest in the present study was in the uncertainty brought on by retrieval failure.

\section{Results and discussion}

The primary interest in the present study was in participants' ratings of their inclination to gamble among trials for which the answer was completely unretrieved. Toward this end, we compared trials for which participants reported a TOT state versus for which they reported a non-TOT state when the question went unanswered. For these analyses of interest, all trials for which there was either a full identification on any attempt (e.g., on the first or second attempt or in response to the partial identification prompt) or a correct partial identification on any attempt (e.g., correct first letter, pronunciation, number of syllables) were removed from further consideration. This was the case in all experiments reported here. The rates of complete identification failure of the answers (labeled "unanswered") are reported in Table 1 for all experiments, along with the rates of full identification ("answer identified") and partial identification ("partially identified"). As can be seen here, more than half of the 80 questions went completely unanswered in all of the experiments reported here.

Also, there is no indication of increased identification likelihood across any of our conditions or experiments. (These numbers will not be discussed further, as the reader is referred to Table 1 for these numbers for each experiment.) For Experiment 1, the rate of reporting a TOT state among completely unanswered questions was $18.75 \%(S D=9 \%)$ or an average of 8.11 of them $(S D=4.31)$.

Turning to the data of primary interest - participants' ratings of their inclination to gamble among unanswered questions - as can be seen in Fig. 1, participants rated their inclination to gamble as being higher during reported TOT states than during reported non-TOT states, $t(18)=4.24, S E$ $=.68, p<.001$, Cohen's $d=.97,95 \%$ CI $[1.46,4.33]$. This finding suggests that the TOT positivity bias is not limited to judgments about the unretrieved target information. The TOT positivity bias extends to concurrent decisions, or unrelated judgments being given around the time of the reported TOT state.

Additionally, as has been shown in previous research (Cleary, 2018), participants exhibited a retrieval bias such that they reported a greater inclination to gamble when target retrieval succeeded and the answer was identified $(M=6.37, S D$ $=3.16$ ) compared with when it failed and the question was unanswered $(M=1.87, S D=1.27), t(18)=5.99, S E=.75, p$ $<.001$, Cohen's $d=1.37,95 \%$ CI [2.92, 6.08]. Cleary (2018) suggests that the retrieval bias reflects use of a fluency heuristic (e.g., Hertwig, Herzog, Schooler, \& Reimer, 2008) whereby ease of retrieval of a piece of information is used as a gauge in making other judgments. However, the present study is novel in showing that ease of retrieval may be used to make completely unrelated judgments that happen to occur around the time of the retrieval access.

\section{Experiment 2}

Experiment 1 demonstrated that the TOT positivity bias is not limited to judgments about the unretrieved information; it extends to concurrent but completely unrelated judgments. Experiment 2 aimed to assess the concurrent nature of this apparent spillover effect. If indeed the apparent bias is a spillover from the warm glow of the TOT experience, it should be necessary to catch people in the initial moments that they are in the grip of the TOT feeling in order for the spillover bias to emerge. To examine this idea, in Experiment 2, the same task was used as in Experiment 1, but this time comparing two conditions. In one condition, participants were prompted to rate their inclination to gamble immediately after indicating the presence or absence of a TOT state (identically to as in Experiment 1). In the other condition, participants were presented with a 10-second delay in between indicating the presence or absence of a TOT state and rating their inclination to gamble. The purpose of this comparison was to test the hypothesis that in the delay condition, the TOT positivity bias 
Table 1 Mean number of questions answered, partially answered, and completely unanswered (out of 80)

\begin{tabular}{|c|c|c|c|c|c|c|}
\hline \multirow[t]{2}{*}{ Experiment/Condition } & \multicolumn{2}{|c|}{ Answer Identified } & \multicolumn{2}{|c|}{ Partially Identified } & \multicolumn{2}{|c|}{ Unanswered } \\
\hline & $M$ & $S D$ & $M$ & $S D$ & $M$ & $S D$ \\
\hline Experiment 1 (Rating) & 32.74 & 11.80 & 3.21 & 2.10 & 44.05 & 12.12 \\
\hline Experiment 2 (rating, no delay & 30.78 & 10.56 & 2.71 & 1.72 & 46.52 & 10.85 \\
\hline Experiment 2 (rating, delay) & 28.08 & 11.32 & 2.49 & 1.69 & 49.43 & 11.38 \\
\hline Experiment 3 (rating \& behavior) & 28.10 & 10.53 & 2.72 & 2.24 & 49.18 & 10.20 \\
\hline
\end{tabular}

would be significantly diminished if it is indeed the result of a fleeting positive feeling.

\section{Method}

Participants Participants were 186 Colorado State University students who each participated in exchange for credit in a course. The reason for the higher sample size than in Experiment 1 was because we were seeking evidence of a between-subjects interaction, rather than a simple effect obtainable via a paired-samples $t$ test. Six participants were lost due to reporting no TOTs and thus were not included in the analyses reported for this experiment. Among the remaining participants, half (90) had been randomly assigned to the delay condition, and half (90) had been randomly assigned to the standard (nondelay) condition.

Materials Stimuli were the same general-knowledge questions and target answers as in Experiment 1.

Procedure The procedure was identical to that used in Experiment 1, with the exception that participants were randomly assigned to one of two versions in a between-subjects design. One version was identical to the procedure used in Experiment 1 (thus, serving as a replication). The other version was identical, except for the insertion of a 10-s delay between the TOT prompt and the prompt to rate the inclination to gamble. During this delay between prompts, the screen said, "Please wait. . . . More questions coming momentarily."

\section{Results and discussion}

Before turning to the data of primary interest (the ratings of the inclination to gamble as a function of reported TOT versus non-TOT states), it is important to consider the rates of reporting TOT states. Participants' rates of reporting TOT states among unanswered questions in Experiment 2 were comparable to the rate reported in Experiment 1. Moreover, in Experiment 2 there was no significant difference between TOT rates in the no-delay $(M=.18, S D=.10)$ or the delay $(M$ $=.21, S D=.12)$ conditions, $t(178)=1.68, S E=.02, p=.10$.

The primary interest was in participants' ratings of their inclination to gamble among trials for which the question went unanswered, comparing trials for which they reported a TOT state versus a non-TOT state as a function of the delay condition. As in Experiment 1, this meant that the primary interest

\section{Mean Ratings of the Inclination Toward Gambling}

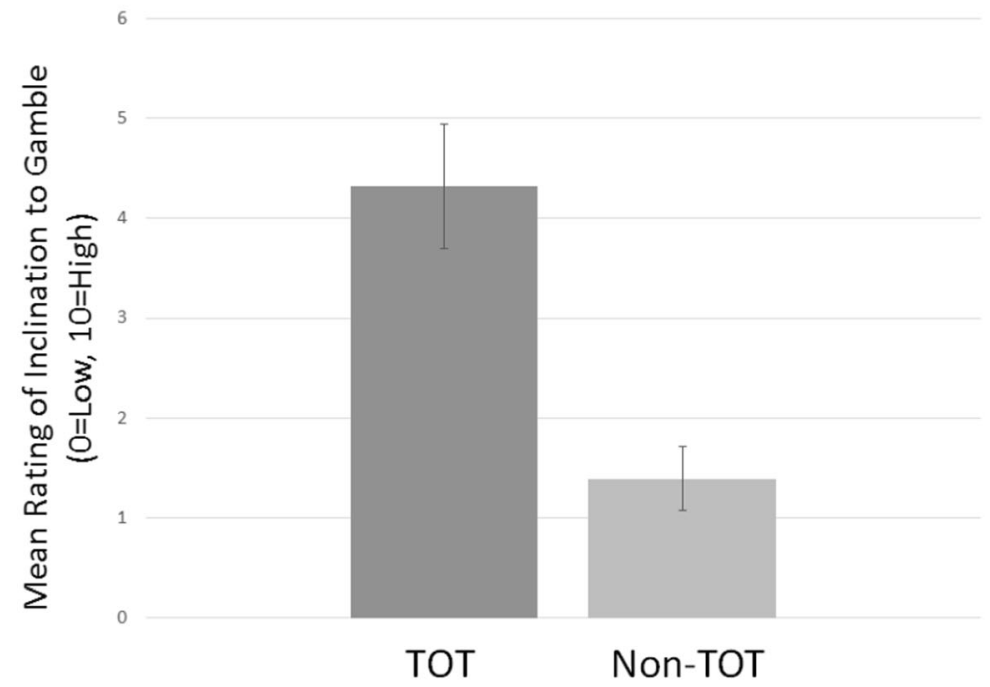

Fig. 1 Mean ratings of participants' inclination to gamble as a function of reported TOT versus non-TOT state 
was in trials for which retrieval failed on the first, second, and partial attempt (the unanswered trials in Table 1). A $2 \times 2$ TOT status (TOT state vs. non-TOT state) $\times$ delay condition (no delay vs. 10-s delay) mixed ANOVA on inclination to gamble ratings revealed several findings. First, as can be seen in Fig. 2 , participants rated their inclination to gamble as being higher during reported TOT states than during reported non-TOT states, as shown by a main effect of TOT status, $F(1,178)=$ 95.62, MSE $=1.90, p<.001, \eta_{\mathrm{p}}{ }^{2}=.35$. This replicates the pattern shown in Experiment 1.

Second, and important to the primary goal of Experiment 2, there was a significant interaction such that the TOT bias effect was larger in the no-delay than in the delay condition, $F(1,178)=6.36, M S E=1.90, p=.01, \eta_{\mathrm{p}}{ }^{2}=.03$, This finding supports the hypothesis that the apparent spillover effect of the TOT positivity bias coincides with the TOT experience itself. If even 10 seconds pass between the prompt regarding the TOT state and the prompt to make an unrelated decision (in this case, rating the inclination to take a gamble), the bias to be inclined to take a gamble diminishes. Though significantly diminished, the bias effect was still present, as shown by a paired-samples $t$ test for just those judgments given by participants in the delay condition, $t(89)=5.84, S E=.18, p<.001$, Cohen's $d=.62,95 \%$ CI [.70, 1.41]. Finally, a main effect of delay condition also emerged from the $2 \times 2$ ANOVA, $F(1$, 178) $=10.15, M S E=4.83, p=.002 ., \eta_{\mathrm{p}}{ }^{2}=.05$. This main effect was such that ratings of the inclination to gamble were lower overall in the delay condition than in the non-delay condition.

As in Experiment 1, participants in Experiment 2 exhibited a bias toward a greater inclination to take an unrelated gamble when target retrieval succeeded compared with when it failed. A $2 \times 2$ target retrieval status (target identified vs. question unanswered) $\times$ delay condition (no delay vs. 10-s delay) mixed ANOVA revealed a main effect of target retrieval status, $F(1,178)=464.52, M S E=4.64,, p<.001, \eta_{\mathrm{p}}{ }^{2}=.72$. Mean inclination to gamble ratings were higher during instances of successful target identification both when there was no delay $(M=6.92, S D=2.50)$ and when there was a delay $(M=6.61, S D=2.50)$ than when the question went unanswered both when there was no delay $(M=2.09, S D=$ $1.63)$ and when there was a delay $(M=1.66, S D=1.32)$. There was no interaction from this analysis $(F<1.0)$. However, a possible main effect of delay condition was approaching significance, $F(1,178)=3.23, M S E=3.82, p=$ $.074, \eta_{\mathrm{p}}{ }^{2}=.02$; this was such that participants may have been slightly less inclined to gamble after a delay overall, but this was not the focus our study, and the retrieval bias itself persisted across the delay nonetheless.

\section{Experiment 3}

Experiments 1 and 2 suggest that the TOT positivity bias spreads to other decisions not related to the sought-after target or to the process of seeking the target. It is unclear how far this spillover effect spreads. It could be the case that the TOT positivity bias only affects hypothetical decisions that do not actually result in risks taken. However, there is evidence to suggest that priming a positive mindset, such as by cuing participants to remember past wins, increases actual gambling behavior (Ludvig et al., 2015). Therefore, it is plausible that the positive-oriented mindset that seems to coincide with the onset of the TOT state will prime actual gambling behavior. Experiment 3 was aimed at examining this. Toward this end, participants not only rated their inclination to gamble but also had the opportunity to take a gamble where they would win or lose points upon doing so. Because there is no precedent for such a behavioral effect, there was no basis on which to compute a power analysis (other than to prior TOT bias effects found with ratings). Thus, we aimed for a higher sample size $(N=50)$ than in Experiment 1 in order to ensure unambiguous detection of a potential behavioral effect, should one be there, but a sample size that was also comparable with sample sizes

\section{Mean Ratings of the Inclination Toward Gambling}

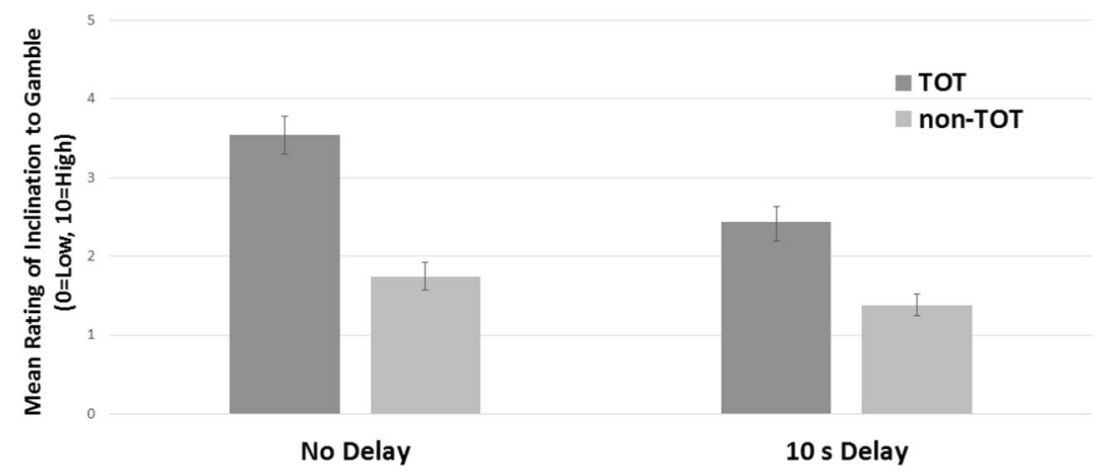

Fig. 2 Mean ratings of participants' inclination to gamble as a function of reported TOT versus non-TOT state, and whether there was a delay between the TOT report and the prompt to rate the inclination to gamble 
used with other TOT bias effects found with ratings (e.g., Cleary \& Claxton, 2015, who used 40-56 participants per experiment).

\section{Method}

Participants Fifty-two Colorado State University undergraduates participated in exchange for course credit. One was lost due to a computer crash, and one was removed that did not report any TOT states, leaving a total of 50 participants.

Materials The same general-knowledge questions and answers from Experiments 1 and 2 were used.

Procedure Participants followed the same procedure outlined in Experiment 1, with a few exceptions. At the beginning of the experiment, participants were also told that they would be asked to decide whether to bet on the coin flip by pressing $H$ for betting heads, $T$ for betting tails, or $N$ if they did not want to gamble on that trial. Participants were told that they would start with 100 points, and that guessing correctly would gain them 10 points, while guessing incorrectly would lose them 10 points. Choosing not to gamble would mean their points were not affected. During the experiment, an image of two quarters remained on the screen-one showing heads and the other showing tails. Following the prompt to rate the inclination to gamble, the prompt to choose $H, T$, or $N$ (heads, tails, or not gambling) was presented. If participants chose to gamble on a given trial (by pressing $H$ or $T$ ), they were immediately shown the random outcome of the "coin flip" (a computer-generated heads or tails outcome with a .50 probability of either result) with a statement on whether they won or lost, and their updated points balance. The 80 trials were split into two blocks. Upon completion of the first test block, participants' points were reset to 100 for the second test block. This was done so participants would not feel compelled to stop gambling if they had won or lost a lot of points in the first block.

\section{Results and discussion}

As with Experiments 1 and 2, it is important to first consider the rate of reporting TOT states before turning to the data of primary interest. Among unanswered questions, participants in Experiment 3 reported TOT states at a mean rate of .16 (SD $=.09$ ).

Turning to the data of interest, first, we examined the inclination to gamble ratings. In replication of Experiments 1 and 2, participants gave higher ratings of inclination to gamble in TOT states $(M=4.36, S D=2.55)$ than in non-TOT states $(M=$ $2.58, S D=2.40), t(49)=6.22, S E=.29, p<.001$, Cohen's $d=$ $0.72,95 \%$ CI [1.21, 2.36].
Turning to our primary question in Experiment 3, participants were also more likely to actually gamble during TOT states, as shown by a higher probability of choosing either $H$ or $T(M=.68, S D=.33)$ than during non-TOT states $(M=.48$, $S D=.30), t(49)=4.88, S E=.04, p<.001$, Cohen's $d=0.62$, $95 \%$ CI $[.12, .28]$. Thus, the increased inclination to gamble during TOT states translated to an actual increase in gambling behavior during TOT states. In short, participants do not simply report a higher inclination to gamble when in a TOT state but are also more likely to take a gamble.

Finally, the retrieval bias was also replicated in Experiment 3 , such that participants showed a higher inclination to gamble after answer identification occurred $(M=5.88, S D=2.70)$ than when the question went unanswered $(M=2.85, S D=$ 2.32) $t(49)=7.80, S E=.39$, Cohen's $d=1.20, p<.001,95 \%$ CI $[2.25,3.81]$. The retrieval bias also extended to the actual decision to gamble. Participants were more likely to gamble when they had succeeded with an answer identification $(M=$ $.81, S D=.23)$ than when they left the question unanswered $(M$ $=.51, S D=.29), t(49)=7.69 . S E=.04$, Cohen's $d=1.30, p<$ $.001,95 \%$ CI $[.22, .38]$. In short, participants are more likely to take a gamble following successful retrieval of an answer than following an inability to answer.

\section{General discussion}

\section{Overview of the present study}

The tip-of-the-tongue (TOT) state bias is a tendency for judgments during the uncertainty of retrieval failure to differ depending on whether a TOT state or a non-TOT state is reported (e.g., Cleary, 2006; Cleary, 2018; Cleary \& Claxton, 2015; Cleary et al., 2014). The present study focused on a particular manifestation of the TOT state bias, referred to here as the TOT positivity bias, which is an inclination toward inferring positive qualities or characteristics of the unretrieved information when in a TOT state compared with when not (Cleary, 2018). The present study examined whether the TOT positivity bias spills over into concurrent unrelated decisions that happen to occur around the time of the TOT. Toward this end, a concurrent task completely unrelated to searching for a word was used: Making a decision about risk-taking involving gambling. This task allowed for an assessment of potential spillover effects into both unrelated concurrent judgments and actual behavior itself. We found that the TOT positivity bias extended to an unrelated decision such that participants felt more inclined during TOTs to take an unrelated gamble when prompted soon after. This spillover effect was largely concurrent with the TOT experience, as the increased inclination to want to gamble faded after a delay of 10 seconds between the TOT report and the prompt to rate the inclination to gamble. Finally, the spillover effect of the TOT positivity bias affected 
actual gambling behavior itself: Participants were more likely to gamble for points immediately following TOT reports than non-TOT reports.

\section{Implications for two separate phenomena}

The present findings have relevance for informing understanding of two separate phenomena. The first is the etiology of the TOT experience and its emotional components. The second is the role of priming of momentary mindset in gambling inclinations and behavior.

\section{Implications for TOT etiology and its emotional components} Starting with the first-TOT etiology and its emotional components - the fact that there appears to be an initial fleeting positive-seeming emotional component to the TOT provides some counterintuitive clues as to the etiology of the TOT experience itself. Though the causal directionality of the association between the fleeting positive feeling (the purported "warm glow") and the TOT experience itself is not yet clear, the fact that the association is there provides a potential clue about the feelings that accompany the TOT experience. For example, one possibility is that the feeling of being in a TOT state is exactly that - a feeling. Does the TOT state happen first, followed by the fleeting "warm glow" feeling in response? Or does the TOT state follow the fleeting "warm glow" feeling such that the "warm glow" feeling is itself the signal indicating the presence of a TOT state in the first place? Future research should further examine this question. However, regardless of the direction of causality, a potential clue to the emotional components of the TOT state comes from the fact that the associated valence appears to be positive, and that the positive disposition or mindset that accompanies the positive valence influences concurrent decisions.

It may seem counterintuitive that TOTs would be associated with positive, rather than negative, valence. However, there are clues apparent in the literature for why this might be expected. First, Metcalfe et al. (2017) found that TOTs are associated with increased curiosity and motivation to use limited resources to discover the as yet unknown answer. Second, Gollan and Brown (2006) presented reasons to believe that TOTs are better viewed as instances of retrieval success rather than failure. Taken together, these results suggest that TOTs may be experienced as positive insofar as they are indications of the presence of potentially relevant information in the experiencer's knowledge base as opposed to knowing nothing relevant. Thinking that the answer is there in one's knowledge base may feel positive compared with feeling that one does not know the answer at all, and feeling that it is there may be motivating in terms of putting forth continued effort toward pursuing that sought-after answer.
Fitting with the idea that TOTs may be felt as positive because they indicate a form of retrieval success, full retrieval success itself (correctly identifying the target answer) is associated with a positivity bias as well. This was shown by Cleary (2018) as well as in the present study. In the present study, the retrieval bias exhibited spillover effects into the unrelated decisions about gambling and the gambling behavior itself. In fact, the retrieval bias is typically a larger-magnitude version of the TOT positivity bias, including in the present study. This points toward the possibility that full-blown retrieval success is experienced as very positive affectively, and this leads to spillover effects to other decisions, whereas TOTs - perhaps a partial form of retrieval success-are experienced as more positive than no success at all, but less positive than fullblown retrieval success. Overall, this pattern fits with the claim that TOTs are best viewed as a form of retrieval success rather than failure (Gollan \& Brown, 2006). In this way, the present results inform theory regarding TOT etiology and emotional components.

\section{Implications for priming effects in gambling inclinations and} behavior The fact that the positive-seeming affect surrounding the TOT experience spills over into the risk-taking decisions and behavior involved in gambling has implications for the study of risk-taking itself. Previous research has examined the effects of priming on gambling, and from this work is it known that priming a positive mindset toward gambling, such as by cuing memories of prior wins, increases gambling behavior (Ludvig et al., 2015). While there are indications in the literature that positive affect is associated with more reckless gambling, such as the finding of a positive correlation between positive affect and reckless betting behavior (e.g., Cummins, Nadorff, \& Kelly, 2009), to our knowledge, no studies have yet shown the kind of spillover effects reported in the present study. Somewhat similar to the halo effect (e.g., Morewedge \& Kahneman, 2010), the patterns reported in the present study seem to suggest that mere concurrence with the afterglow of an unrelated positive-seeming event can influence risk-taking inclinations and behavior. In the present study, the afterglow affecting gambling decisions and behavior happen to be coming from the positivity associated with TOTs, but it is likely that any positive-seeming experience can elicit the same type of afterglow and spillover effects shown with TOTs. In support of this idea, we show in the present study a retrieval bias as well with respect to decisions to gamble. When people succeed at fully retrieving the target answer to a question, they feel more inclined to gamble, and they actually do gamble more often - even more often than just during TOTs. This suggests that any momentarily positiveseeming experience can spill over into a completely unrelated concurrent decision involving risk. Thus, anything that primes a person to feel momentarily positive might also prime an increased inclination toward risk-taking. 


\section{Future directions}

The three experiments reported here pave the way for many interesting possible future lines of research. For example, future research can extend the generality of the spillover effects reported here with other unrelated tasks besides a simple gambling task. Some possible unrelated task types include stock market decisions (which may be akin to gambling), or choosing to trust a pictured person with something important, such as caring for a pet, or choosing to take on a difficult challenge versus pass on it. There are likely many more possible unrelated tasks that could be examined in search of TOT positivity bias spillover effects.

Another potentially fruitful future direction concerns the question of why, anecdotally, many people associate TOTs with frustration rather than positivity. We hypothesize that participants' affective experience might reverse across a long time trajectory following the initial TOT response. That is, while the affective experience might initially be positive in the early moments of the TOT onset (prompting curiosity or excitement), it may be that over time (if the answer is not discovered) the affective experience turns to frustration. Our testable hypothesis on this issue is that positivity is the valence that is experienced initially upon TOT onset, and that if the word is successfully found, the experienced valence remains positive (or possibly becomes even more positive). However, if after the passage of the time the target word is not retrieved, the valence changes to negative, as frustration at not finding the target word sets in. What people may tend to remember over time is the frustration associated with never discovering the word rather than the feeling experienced at the time of the TOT onset. This is a testable hypothesis, and we are currently working on testing it in our lab by comparing the experienced valence at the time of the initially reported TOT state, then again following a 30 -second delay after the word remains unretrieved as compared with when the word successfully came to mind during that interval.

Related to the above idea, another testable hypothesis is that successfully retrieving the target answer following an initial TOT will lead to increased positivity, whereas continuing to fail to retrieve it will lead to negativity. We did not design our current experiments to examine this question in the present study, but are currently investigating this question in a new line of work in our lab. We are finding in this line of work that it is a difficult question to address because instances of successful target retrieval after initial retrieval failure are rare, even after a 30 -second delay between the initial TOT report and the time to try again to identify the target answer. Thus, this will need to be a highpowered investigation. Some might wonder if the 10second delay condition in the present study lent itself to additional opportunity for retrieval of the target in a way that might allow for preliminary investigation of the question of whether there is increased positivity when retrieval success happens after initial failure. However, as can be seen in Table 1, there was not an increase in overall identifications after the 10-second delay as compared with the immediate condition. This is consistent with our ongoing work that suggests that successful retrievals after initial failure are rare enough that they are difficult to study in terms of any associated biases and feelings.

Another question for future research concerns what time delay would be required for the complete disappearance of the TOT positivity bias and its spillover effects. In the present study, although the spillover effects of the TOT positivity bias significantly diminished after a 10 -second delay, the bias was still present. Thus, it is a worthwhile question how long it would take for it to completely disappear. Future research should examine this question. We are also currently examining this question in our lab by using longer time delays (30 seconds instead of 10). On a related note, it would also be interesting to examine whether TOT reports themselves decrease in likelihood after a delay. The present study was not designed to examine this, as the TOT prompt happened immediately following the attempt at answering the question. Future research should examine whether TOTs themselves diminish after a delay between the question and the prompt to indicate the presence versus the absence of a TOT.

Finally, it might also be interesting to examine individual differences in the types of spillover effects presented here. For example, some people may be averse to gambling or risktaking in general, and these individual differences among people might interact with the spillover effects shown in the present study. For example, some people may be more susceptible to these effects than others, and it could be worthwhile to investigate if this is the case and if so, what differentiates people along these lines.

Open practices statement None of the experiments reported here were preregistered, but the data are available at the Open Science Framework OSF Link: https://osf.io/t75jr/

\section{References}

Brown, A. S. (1991). A review of the tip-of-the-tongue experience. Psychological Bulletin, 109, 204-223.

Brown, A. S. (2012). The tip of the tongue state. Hove, England: Psychology Press.

Brown, R., \& McNeill, D. (1966). The "tip of the tongue" phenomenon. Journal of Verbal Learning and Behavior, 5, 325-337.

Burke, D. M., MacKay, D. G., Worthley, J. S., \& Wade, E. (1991). On the tip of the tongue: What causes word finding failures in young and older adults? Journal of Memory and Language, 30, 542-579.

Cleary, A. M. (2006). Relating familiarity-based recognition and the tipof-the-tongue phenomenon: Detecting a word's recency in the absence of access to the word. Memory \& Cognition, 34, 804-816. 
Cleary, A. M. (2018). The biasing nature of the tip-of-the-tongue experience: When decisions bask in the glow of the tip-of-the-tongue state. Journal of Experimental Psychology: General. Advance online publication. doi:https://doi.org/10.1037/xge0000520

Cleary, A. M., \& Claxton, A. B. (2015). The tip-of-the-tongue heuristic: How tip-of-the-tongue states confer perceptibility on inaccessible words. Journal of Experimental Psychology: Learning, Memory, and Cognition, 41, 1533-1539.

Cleary, A. M., Konkel, K. E., Nomi, J. N., \& McCabe, D. P. (2010). Odor recognition without identification. Memory \& Cognition, 38, 452460.

Cleary, A. M., \& Reyes, N. L. (2009). Scene recognition without identification. Acta Psychologica, 131, 53-62.

Cleary, A. M., \& Specker, L. E. (2007). Recognition without face identification. Memory \& Cognition, 35, 1610-1619.

Cleary, A. M., Staley, S. R., \& Klein, K. R. (2014). The effect of tip-ofthe-tongue states on other cognitive judgments. In B. L. Schwartz \& A. S. Brown (Eds.), Tip-of-the-tongue states and related phenomena (pp. 75-94). Cambridge, England: Cambridge University Press.

Cummins, L. F., Nadorff, M. R., \& Kelly, A. E. (2009). Winning and positive affect can lead to reckless gambling. Psychology of Addictive Behavior, 23, 287-294.

D’Angelo, M. C., \& Humphreys, K. R. (2012). Emotional cues do not increase the likelihood of tip-of-the-tongue states. Memory \& Cognition, 40, 1331-1138.

Gollan, T. H., \& Brown, A. S., (2006). From tip-of-the-tongue (TOT) data to theoretical implications in two steps: When more TOTs means better retrieval. Journal of Experimental Psychology: General, 135, 462-483.

Hertwig, R., Herzog, S. M., Schooler, L. J., \& Reimer, T. (2008). Fluency heuristic: A model of how the mind exploits a by-product of information retrieval. Journal of Experimental Psychology: Learning, Memory, and Cognition, 34, 1191-1206.

Koriat, A., \& Lieblich, I. (1974). What does a person in a "TOT" state know that a person in a "don't know" state doesn't know. Memory \& Cognition, 2, 647-655.

Ludvig, E. A., Madan, C. R., \& Spetch, M. L. (2015). Priming memories of past wins induces risk seeking. Journal of Experimental Psychology: General, 144, 24-29. doi:https://doi.org/10.1037/ xge 0000046

Metcalfe, J., Schwartz, B. L., \& Bloom, P. A. (2017). The tip-of-thetongue state and curiosity. Cognitive Research: Principles and Implications, 2(31). doi:https://doi.org/10.1186/s41235-017-0065-4

Monin, B. (2003). The warm glow heuristic: When liking leads to familiarity. Journal of Personality and Social Psychology, 85, 10351048.

Morewedge, C. K., \& Kahneman, D. (2010). Associative processes in intuitive judgment. Trends in Cognitive Sciences, 14, 435-440.

Nelson, T. O., \& Narens, L. (1980). Norms of 300 general-information questions: Accuracy of recall, latency of recall, and feeling-of- knowing ratings. Journal of Verbal Learning and Verbal Behavior, $19,338-368$.

Nisbett, R., \& Wilson, T. D. (1977). The halo effect: Evidence for the unconscious alteration of judgments. Journal of Personality and Social Psychology, 34, 250-256.

Payne, B. K., Brown-Iannuzzi, J. L., \& Loersch, C. (2016). Replicable effects of primes on human behavior. Journal of Experimental Psychology. General, 145(10), 1269-1279. doi:https://doi.org/10. 1037/xge0000201

Rastle, K. G., \& Burke, D. M. (1996). Priming the tip of the tongue: Effects of prior processing on word retrieval in young and older adults. Journal of Memory and Language, 35, 586-605.

Ryals, A. J., Reynolds, B., Patton, C., \& Cleary, A. M. (2018, November). Pupil dilation suggests a role of autonomic arousal in tip-of-thetongue state phenomenology. Poster presented at the Annual Meeting of the Psychonomic Society, New Orleans, LA.

Schwartz, B. L. (1999). Sparkling at the end of the tongue: The etiology of tip-of-the-tongue phenomenology. Psychonomic Bulletin \& Review, 6, 379-393.

Schwartz, B. L. (2001). The relation of tip-of-the-tongue states and retrieval time. Memory \& Cognition, 29, 117-126.

Schwartz, B. L. (2002). Tip-of-the-tongue states: Phenomenology, mechanism, and lexical retrieval. Mahwah, NJ: Erlbaum.

Schwartz, B. L. (2008). Working memory load differentially affects tipof-the-tongue states and feeling-of-knowing judgment. Memory \& Cognition, 36, 9-19.

Schwartz, B. L. (2010). The effects of emotion on tip-of-the-tongue states. Psychonomic Bulletin \& Review, 17, 82-87.

Schwartz, B. L., \& Metcalfe, J. (2011). Tip-of-the-tongue (TOT) states: Retrieval, behavior, and experience. Memory \& Cognition, 39, 737749.

Schwartz, B. L., \& Smith, S. M. (1997). The retrieval of related information influences tip-of-the tongue states. Journal of Memory and Language, 36, 68-86.

Schwartz, B. L., Travis, D. M., Castro, A. M., \& Smith, S. M. (2000). The phenomenology of real and illusory tip-of-the-tongue states. Memory \& Cognition, 28, 18-27.

Teasdale, J. D., \& Russell, M. L. (1983). Differential effects of induced mood on the recall of positive, negative and neutral words. British Journal of Clinical Psychology, 22, 163-171.

Young, M. M., Wohl, M. J., Matheson, K., Baumann, S., \& Anisman, H. (2008). The desire to gamble: The influence of outcomes on the priming effects of a gambling episode. Journal of Gambling Studies, 24, 275-293.

Publisher's note Springer Nature remains neutral with regard to jurisdictional claims in published maps and institutional affiliations. 